\title{
Log10 Enzyme-Linked Immunosorbent Assay Unit
}

National Cancer Institute

\section{Source}

National Cancer Institute. Log10 Enzyme-Linked Immunosorbent Assay Unit. NCI

Thesaurus. Code C68878.

A log arithmic-scale (base 10) unit for measuring concentration and/or reactivity of a test substance (an antigen or antibody of interest) as defined in the literature reference for the particular quantitative enzyme-linked immunosorbent assay method. 